\title{
Technè
}

La science au service de l'histoire de l'art et de la préservation des biens culturels

46 | 2018

Science et conservation

\section{Sous le pinceau de Paul Helleu (1859-1927) : étude d'un fonds de peintures à la faveur de sa restauration}

The brushwork of Paul Helleu (1859-1927): study of a painting collection as a result of its restoration

\section{Virginie Trotignon-Aubert et Élise Cambreling}

\section{(2) OpenEdition}

\section{Journals}

Édition électronique

URL : http://journals.openedition.org/techne/328

DOI : $10.4000 /$ techne.328

ISSN : 2534-5168

Éditeur

C2RMF

Édition imprimée

Date de publication : 1 décembre 2018

Pagination : 23-29

ISBN : 978-2-11-152829-1

ISSN : $1254-7867$

\section{Référence électronique}

Virginie Trotignon-Aubert et Élise Cambreling, « Sous le pinceau de Paul Helleu (1859-1927) : étude d'un fonds de peintures à la faveur de sa restauration », Technè [En ligne], 46 | 2018, mis en ligne le 19 décembre 2019, consulté le 23 juillet 2020. URL : http://journals.openedition.org/techne/328 ; DOI https://doi.org/10.4000/techne.328

\section{(c) (†) $\odot$}

La revue Technè. La science au service de l'histoire de l'art et de la préservation des biens culturels est mise à disposition selon les termes de la Licence Creative Commons Attribution - Pas d'Utilisation Commerciale - Pas de Modification 4.0 International. 
Virginie Trotignon-Aubert Élise Cambreling

\section{Sous le pinceau de Paul Helleu (1859-1927) : étude d'un fonds de peintures à la faveur de sa restauration}

The brushwork of Paul Helleu (1859-1927): study of a painting collection as a result of its restoration
Résumé. Devenu musée Bonnat-Helleu en 2011, le musée des Beaux-Arts de Bayonne se consacre notamment à l'étude et à la promotion de l'œuvre de Paul Helleu. Les peintures de l'artiste, méconnues, forment un ensemble de 39 tableaux, qui ont fait récemment l'objet d'une importante campagne de restauration. En effet, le geste d'Helleu tendait à disparaître sous des interventions à la chronologie confuse : retouches et repeints, vernis trop brillant et encrassé, toiles pliées, coupées et déformées, auvres rentoilées. Les opérations menées en 2015 ont fourni l'occasion de confronter les sources historiques, littéraires et matérielles pour mieux cerner le travail du peintre. Le dialogue et la collaboration entre l'équipe scientifique du musée et les restaurateurs ont permis d'apprécier les traitements à opérer pour restituer sa cohérence à un ensemble hétérogène.

Mots-clés. Bayonne, Paul César Helleu, peinture, restauration, collection.
Abstract. Renamed the Musée Bonnat-Helleu in 2011, the Musée des Beaux-Arts, Bayonne, is notably devoted to the study and promotion of Paul Helleu's works. This collection of thirty-nine little-known paintings has recently undergone a major restoration campaign. Helleu's actual brushwork had, in fact, almost disappeared beneath previous chronologically confused restorations: amendments and repainting; dirt-engrained and overly glossy varnish; folded, cropped and misshapen canvases; remounted works. The restoration programme carried out in 2015 provided an opportunity to compare historical, literary and material sources so as to better comprehend the painter's work. Dialogue and collaboration between the museum's scientific team and the restorers enabled us to decide on which treatments to attempt in order to restore the coherence of this heterogeneous collection.

Keywords. Bayonne, Paul César Helleu, painting, restoration, collection.
En 2009, le musée Bonnat reçoit le legs de Paulette HowardJohnston (Paris, 1904-Biarritz, 2009), fille cadette de l'artiste Paul César Helleu (Vannes, 1859-Paris, 1927), soit plus de 330 biens patrimoniaux dont environ $40 \%$ de tableaux, estampes et dessins de ce dernier. Ils s'ajoutent à ceux déjà conservés depuis 1989, fruits des dons et legs de ses descendants. L'acceptation de cette libéralité par la ville de Bayonne implique le changement de nom de l'établissement, qui devient musée Bonnat-Helleu, musée des Beaux-Arts de Bayonne par un arrêté du 12 mai 2011. Essentiellement connu comme graveur des élégantes et dessinateur aux trois crayons, Paul Helleu fut également peintre. Cette production, que lui-même mésestimait, reste très méconnue en raison de sa dispersion, la plupart des tableaux se trouvant toujours en mains privées.

Dans le cadre de la valorisation du fonds conservé à Bayonne, la restauration de 39 œuvres à l'huile, dans un état hétérogène, est apparue comme une priorité pour saisir toute la complexité de l'œuvre de cet artiste. Cependant, comment mener les interventions dans le respect de la technique originale et en retrouvant la cohérence du fonds ? L'équipe scientifique du musée Bonnat-Helleu s'est heurtée à la pauvreté des connaissances et des sources pouvant guider les choix à opérer. Un dialogue régulier entre les restaurateurs et la conservation a permis de déterminer les partis pris pertinents, au regard de l'histoire du fonds, des quelques éléments connus sur la pratique picturale d'Helleu et des constats réalisés par les restaurateurs. Cette analyse conjointe a fait émerger de nouvelles données concernant tant la technique que l'histoire des tableaux.

\section{Histoire d'une collection}

La première peinture d'Helleu rejoint le musée dès 1989. Six autres y sont déposées par Paulette Howard-Johnston en 1991 et y demeurent jusqu'à intégrer les collections au décès de leur propriétaire. En 1997, Ghislaine de Kermaingant, l'une des petites-filles d'Helleu, lègue pour sa part un grand tableau et 20 œuvres graphiques. Il faut attendre 2010 pour que parviennent à Bayonne 31 autres peintures. Certaines

Virginie Trotignon-Aubert, restaurateur de peintures (v.trotignon.aubert@gmail.com). Élise Cambreling, attachée de conservation du patrimoine, directeur-adjoint du musée Bonnat-Helleu, musée des Beaux-Arts de Bayonne (e.cambreling@bayonne.fr). 

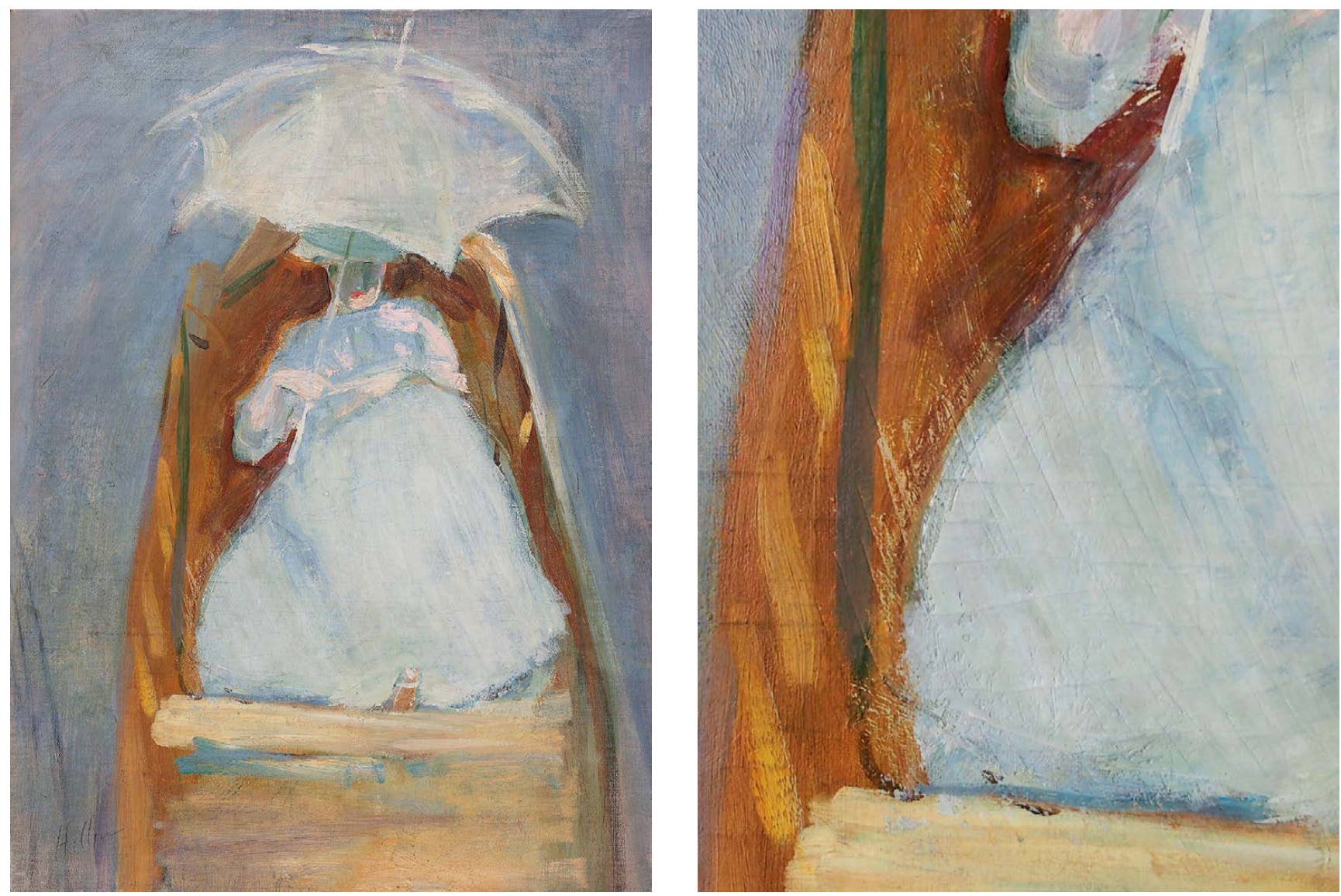

Fig. 1. a. Femme étendue dans une barque, 1888, huile sur toile $(52,5 \times 38,5 \mathrm{~cm})$ (inv. 2010.1.14).

(c) Bayonne, musée Bonnat-Helleu/A. Vaquero.

b. Détail. (C) V. Trotignon-Aubert.

disposent de leur cadre ancien, d'autres sont simplement montées sur châssis. Enfin, deux d'entre elles sont de petits fragments de toile.

En dépit de leur provenance, toutes ne sont pas issues du fonds d'atelier de Paul Helleu. Disparu soudainement en 1927, le peintre laisse à sa veuve, Alice Helleu, un ensemble conséquent de gravures, pastels, dessins et peintures de sa main. L'inventaire dressé après le décès de celle-ci en 1933 en donne un aperçu. S'y trouve, sous le numéro 64, un tableau par Helleu - «Son portrait » qui pourrait être l'autoportrait tardif conservé à Bayonne (inv. CM 472). En revanche, certaines peintures ont transité par le marché de l'art avant d'être achetées par Paulette Howard-Johnston comme Quatre pêches sur un plateau (inv. 2010.1.33), qui fait partie de la succession de Georges Viau ${ }^{1}$. D'autres portent une dédicace et sont donc susceptibles d'avoir appartenu à différents propriétaires. C'est le cas de la marine La Rade de Cowes (inv. CM 557), qui porte la mention «a [sic] l'ami Viau » ou du Vase d'hortensias sur un guéridon carré (inv. 2010.1.27), dédicacé au critique Albert Flament.

Ces œuvres ont donc subi les aléas de conditions de conservation diverses. Plusieurs d'entre elles, léguées par Paulette Howard-Johnston, étaient autrefois accrochées dans l'ancien appartement parisien d'Helleu, et avaient subi un dégât des eaux. Quatorze ont également été stockées dans un coffre de banque, séparées les unes des autres par des rondelles de liège punaisées qui ont marqué et perforé la couche picturale. Globalement, les peintures étaient extrêmement encrassées en raison de leur exposition dans des pièces à vivre. Enfin, des interventions de restauration ont été réalisées sur le support, ainsi que des reprises de la couche picturale qui ont modifié certaines toiles, sans qu'il soit possible de déterminer si leur auteur est l'artiste lui-même ou leur propriétaire ultérieur.

\section{Une peinture confidentielle}

L'histoire du fonds nous renseigne finalement assez peu sur son état. Il n'est pas plus aisé de trouver des informations sur la technique picturale de l'artiste, dont celui-ci a peu parlé de son vivant. Quant à la littérature et à la presse, sources principales d'information, elles nous permettent surtout de comprendre le rapport d'Helleu à son art. Jacques-Émile Blanche le décrit comme un «fanatique de la peinture ", une passion allant avec une exigence qui le pousse parfois à détruire son travail. Il rapporte ainsi les propos de l'artiste, concernant les marines que ce dernier souhaite lui 
emprunter : «Si elles n'ont pas été au poêle ou à la mer, c'est grâce à vous, mon vieil ami. Vous me poussiez à montrer mes tableaux. Trop d'orgueil, et la conscience de l'inutilité de se produire, de nos jours, m'ont empêché d'exposer². » L'épouse du peintre confirme la disparition de nombreuses toiles condamnées par leur auteur, " recouvertes de blanc parce

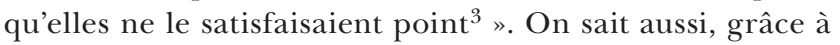
Blanche, que ces peintures étaient conservées « jalousement cachées au public et qu'il [les] donnait, brusquement, à ses amis ${ }^{4} »$. Malgré quelques critiques positives dans les années 1890 , les toiles ne quittent quasiment plus l'atelier lorsque la réputation de pastelliste et graveur mondain d'Helleu est établie.

Les portraits à l'huile sont relativement peu nombreux et prennent essentiellement des proches pour modèle. La figure est également traitée à travers quelques nus féminins ou placée dans un paysage, comme chez les impressionnistes qu'Helleu admire. Cette influence transparaît aussi dans les effets de lumière des marines et des vues urbaines, notamment de cathédrales. La touche fragmentée et la palette chromatique riche en teintes pastel lumineuses sont empruntées à son ami Claude Monet tout comme le travail en plein air que décrit Edmond de Goncourt dans son Journal: « [...] arrive Helleu, qui a passé toute la journée à peindre, par ce froid, les statues de Versailles, à demi ensevelies sous la neige, parlant de la beauté du spectacle, et du caractère de ce monde polaire ${ }^{5}$. » Il produit aussi des natures mortes de fleurs et de fruits, pour lesquelles il adopte des teintes plus automnales, sombres et sourdes.

\section{La matière et l'effet}

Aucune étude n'ayant jamais été menée, l'expertise des restaurateurs sur les 39 œuvres restaurées a fait émerger de nouvelles données techniques concernant l'art d'Helleu. Les tableaux sont de petit format, dépassant rarement le mètre pour la plus grande des dimensions. Cela correspond aux contraintes d'une peinture que l'on devine plutôt intime, souvent pratiquée en extérieur. Les œuvres sont exécutées sur des toiles généralement de contexture fine et serrée ${ }^{6}$, à l'exception d'une huile sur papier marouflé ${ }^{7}$. Riche, la matière picturale, appliquée sur une préparation claire d'aspect industriel, est variable en épaisseur selon la composition et ses motifs. Elle est fine et transparente lorsque la facture est

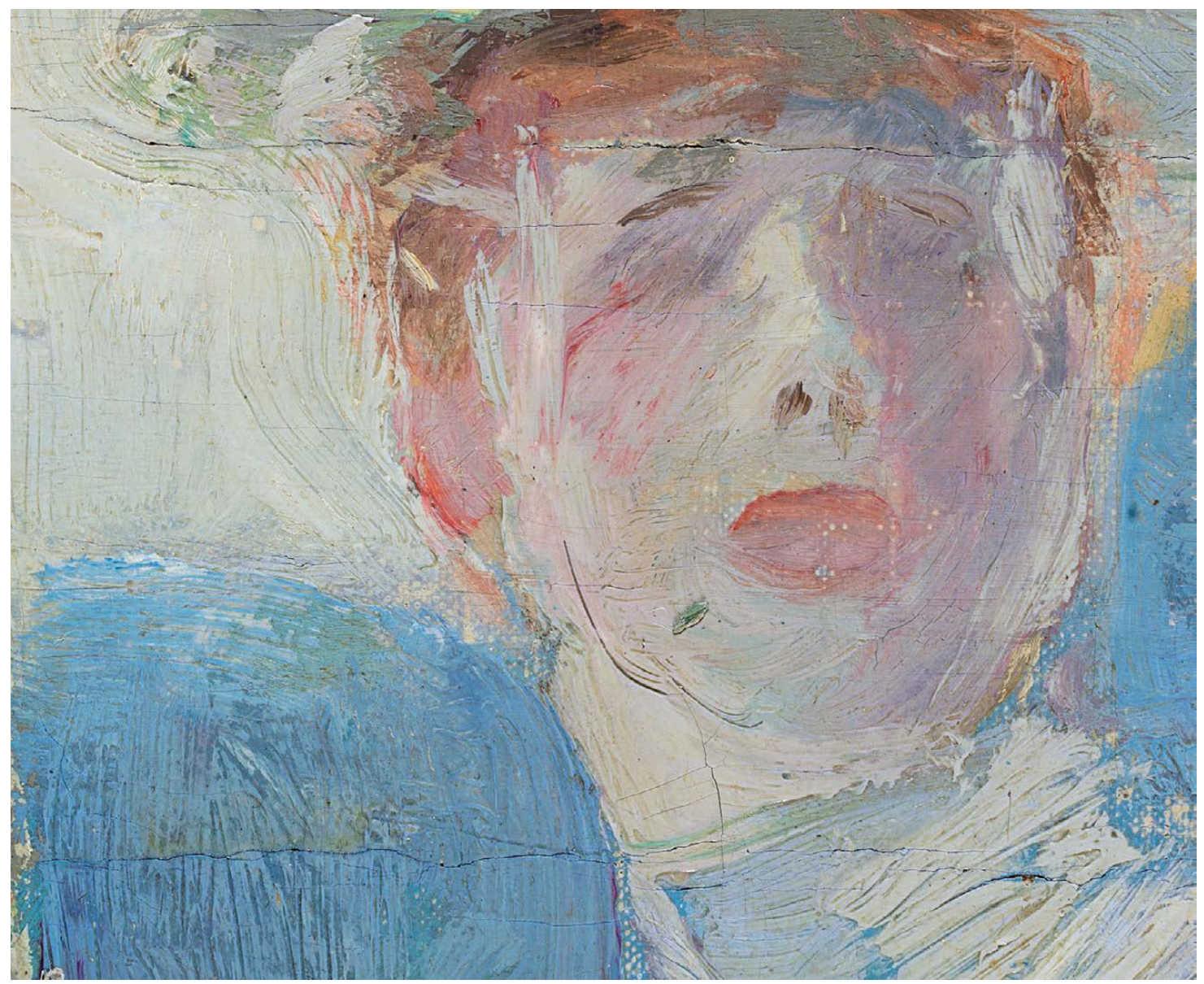

Fig. 2. Alice Helleu à Varengeville, 1887, huile sur toile (71,5 x 38,7 cm) (inv. 2010.1.38), détail. (c) Bayonne, musée Bonnat-Helleu/A. Vaquero. 
rapide, avec des réserves, et comporte fréquemment des empâtements localisés. L'un des tableaux conservés à Bayonne, représentant une femme sur le pont d'un yacht (inv. 2010.1.29), est demeuré inachevé. Un tracé sous-jacent au graphite est perceptible, mais ne paraît pas préparatoire au motif rapidement posé à l'aide de jus colorés. Au contraire, Les Vitraux de l'église Saint-Jacques, Dieppe (inv. 2010.1.47) et l'Autoportrait (inv. CM 472) se distinguent par une forte épaisseur sur la composition entière.

L'écriture de l'artiste témoigne d'un geste vif, nerveux et précis : les fonds sont souvent brossés, et les motifs constitués de touches posées les unes sur les autres, sans modulation, avec des outils qui sont davantage apparentés à des brosses et à des couteaux qu'à des pinceaux doux. Cette technique est courante autour de 1900. Quelques griffures font cependant son originalité et sont probablement à mettre en relation avec l'activité de graveur d'Helleu. En effet, la peinture est rayée nerveusement jusqu'à la préparation, sans doute avec la hampe du pinceau, pour souligner une forme, rendue plus graphique grâce à ce procédé (fig. 1 a et b). Des zones peintes sont également grattées localement : l'artiste «maltraite » la matière afin de lui donner plus de force. En dépit de ce que les témoignages contemporains laissent transparaître, Paul Helleu est sûr de lui quand il peint et retravaille peu ses compositions, ainsi qu'en témoigne la rareté des repentirs.

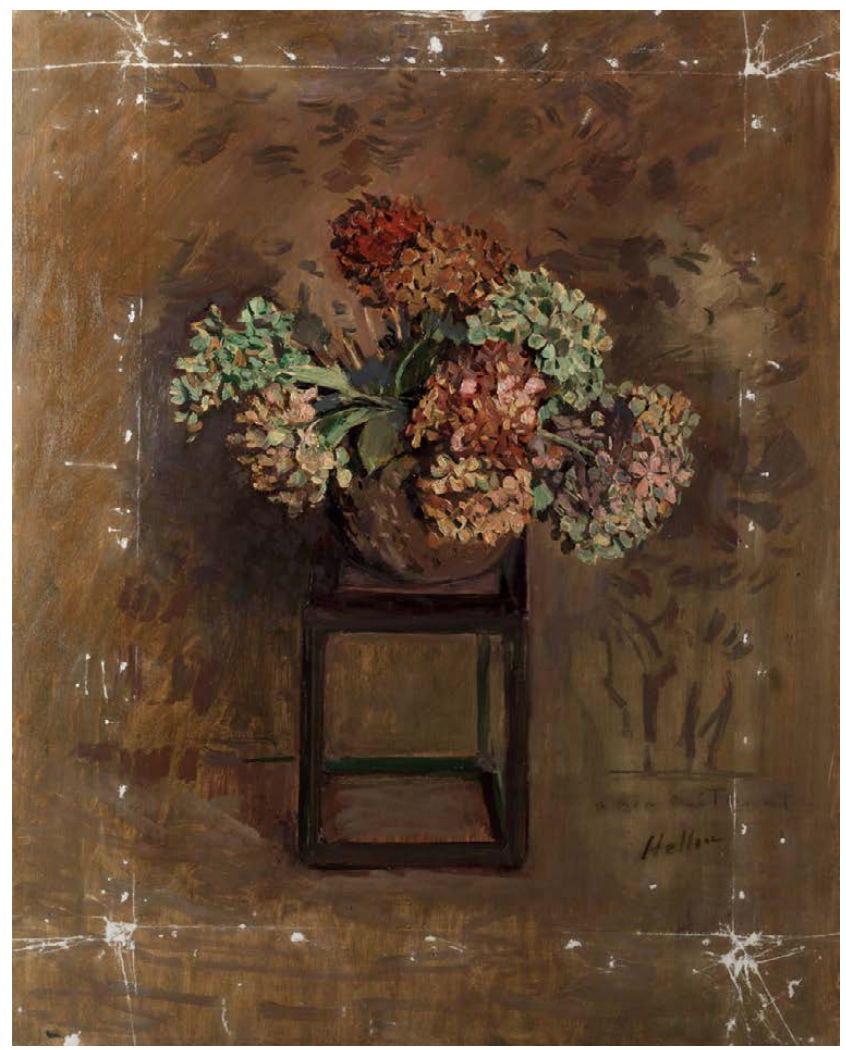

Fig. 3. Vase d'hortensias sur un guéridon carré, huile sur toile $(91,8 \times 72,9 \mathrm{~cm})$ (inv. 2010.1.27).

(c) Bayonne, musée Bonnat-Helleu/A. Vaquero.

\section{Enjeux d'une restauration}

Les constats effectués sur les tableaux mettent en évidence l'hétérogénéité de leur état, parfois lié à de mauvaises conditions de stockage. $46 \%$ des toiles souffrent d'une déformation du support, près de $60 \%$ d'une perte d'adhérence de la couche picturale et $67 \%$ de lacunes. En outre, une partie d'entre elles est affectée par d'anciennes interventions au sujet desquelles nous ne disposons d'aucune information. Trois œuvres ont visiblement été roulées, pliées puis retendues sur châssis, notamment Alice Helleu à Varengeville (inv. 2010.1.38, fig. 2). Seize rentoilages, qui paraissent abusifs, ont écrasé la matière picturale encore trop fraîche ${ }^{8}$. Des repeints dégradés sont présents sur 17 toiles, ainsi que de nombreuses reprises de composition à l'originalité douteuse, décalées chromatiquement, qui ne recouvrent aucune usure. Enfin, dans $38 \%$ des cas, les vernis sont trop présents, parfois très brillants et altérés. L'hétérogénéité de la collection est donc une réalité matérielle et la cohérence de l'ensemble pictural conservé à Bayonne un enjeu majeur de cette campagne de restauration. Cela répond à la volonté du musée de valoriser l'œuvre d'Helleu, conformément aux souhaits de la testatrice, par l'étude, le prêt et l'exposition temporaire ou permanente.

Afin d'assurer un traitement harmonieux du fonds, une même équipe a été retenue pour réaliser l'intégralité des restaurations en 2015 : Virginie Trotignon-Aubert et MarieAnge Laudet-Kraft pour la couche picturale, Jean-François Hulot et Cécile des Cloizeaux pour le support. Plusieurs objectifs ont été menés en concomitance, dans le respect des principes déontologiques : comprendre la technique de Paul Helleu, redonner une unité à cette collection et revaloriser la matière originale, sans intervention irréversible. La façon d'aborder les traitements a fait l'objet d'un questionnement collégial permanent, favorisé par l'intervention des restaurateurs in situ. Les données historiques et les observations concrètes au cours de la restauration s'éclairent mutuellement pour trouver le niveau de traitement adapté, en préservant les intentions de l'artiste. La recherche de la cohérence de l'ensemble du fonds a motivé une homogénéisation des interventions à l'issue du travail.

\section{À la recherche de l'œuvre originelle}

Concernant le support, il est certain que des restaurateurs sont déjà intervenus sur au moins $41 \%$ des œuvres. Le rentoilage, procédé interventionniste fréquemment employé dans les années 1980, ainsi que le type de châssis moderne utilisé, suggère qu'une série d'opérations a été menée il y a une trentaine d'années. Le démontage ou la reprise des rentoilages, encore fonctionnels et bordés de kraft, ne s'avérant ni indiqué, ni souhaitable, le travail s'est donc concentré sur les problèmes d'adhérence ponctuels de la couche picturale à son support. Les soulèvements ont été refixés et la tension révisée afin de résorber les déformations. Les traitements les plus interventionnistes ont consisté à remettre le Vase d'hortensias sur un 


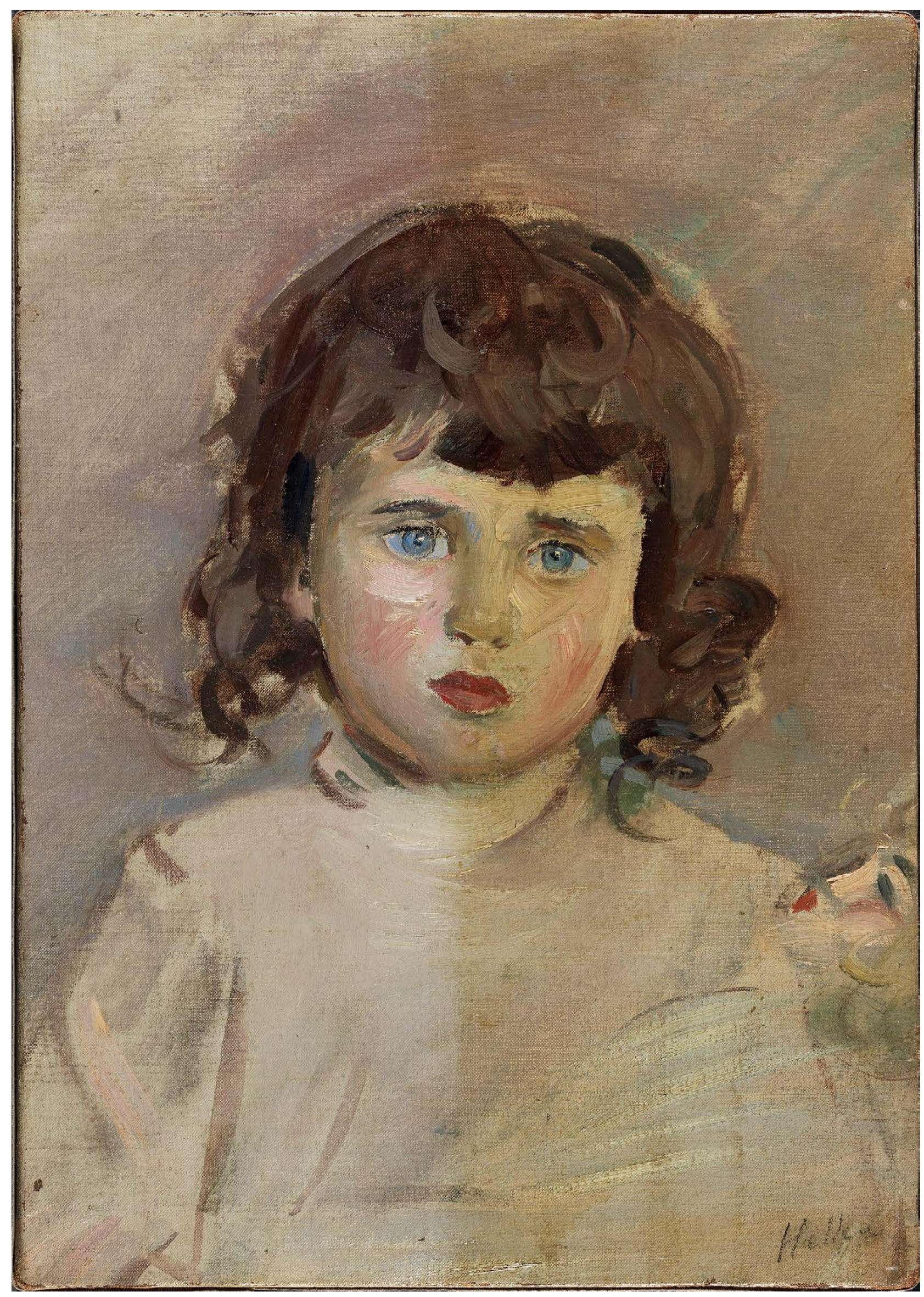

Fig. 4. Ellen Helleu à la poupée, vers 1891, huile sur toile (46,5 x 33,3 cm) (inv. 2010.1.41), en cours de nettoyage. ( ) Bayonne, musée Bonnat-Helleu/A. Vaquero. 

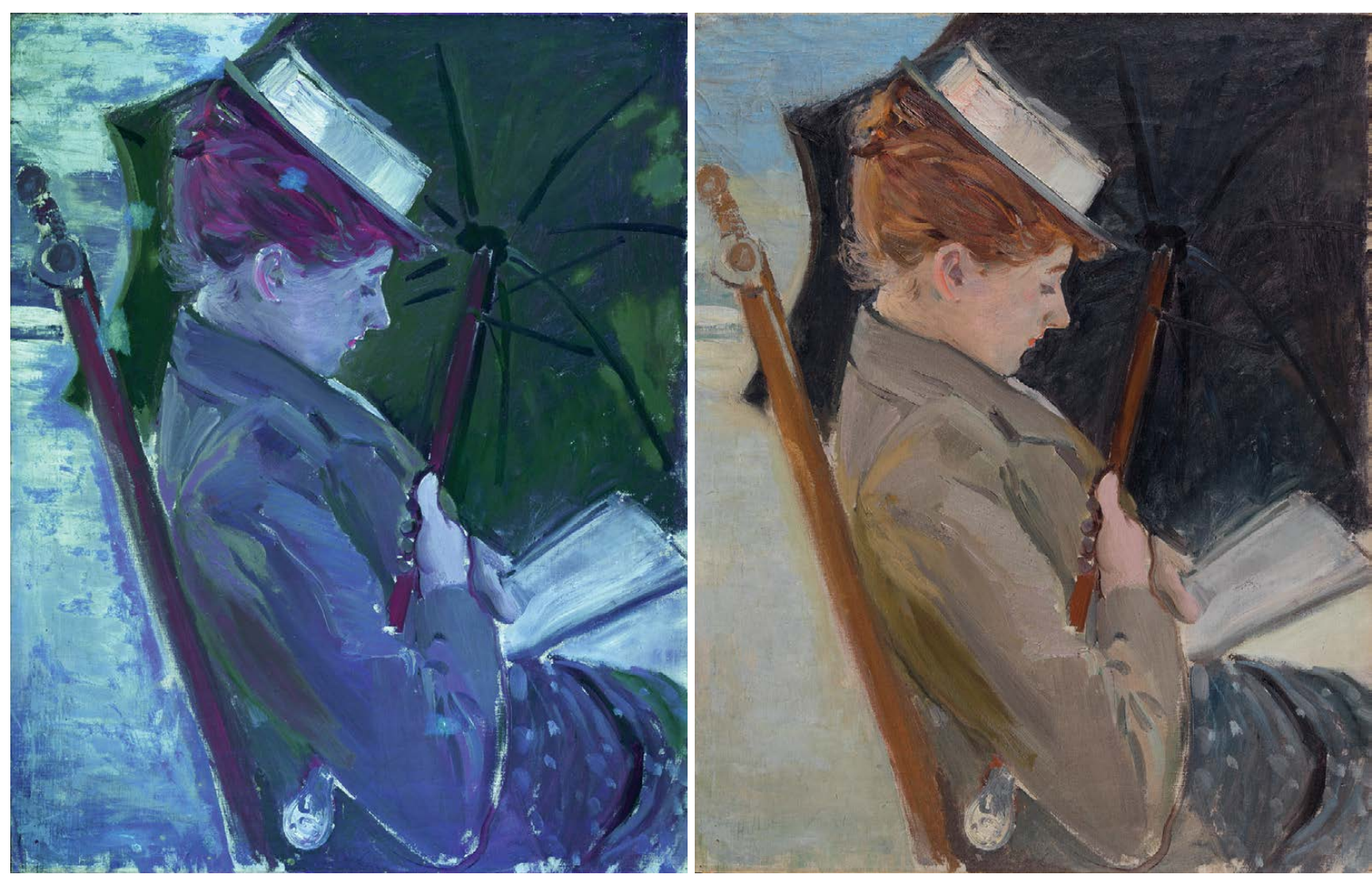

Fig. 5. Alice Helleu lisant sur la plage de Deauville, 1892 ou 1896, huile sur toile (79 x 63 cm) (inv. 2010.1.39). À gauche, sous lumière ultraviolette et à droite, avant restauration. (C) Bayonne, musée Bonnat-Helleu/A. Vaquero.

guéridon carré (inv. 2010.1.27, fig. 3) dans son format original, afin de retrouver la dédicace à Albert Flament, et à changer les supports, inadaptés, des fragments.

Les problèmes esthétiques, hormis les opérations habituelles de décrassage et de retouches sur les lacunes, se sont principalement posés avec la question de la conservation ou non des vernis, présents sur les tableaux. Leur authenticité n'est pas évidente. Le seul exemple de vernis apparemment original est relevé sur Femme étendue dans une barque (inv. 2010.1.14) (fig. 1), qui présente quelques plages d'une matière résineuse correspondant à des rehauts du motif, technique fréquemment observée chez les peintres du début du $\mathrm{xx}^{\mathrm{e}}$ siècle qui vernissent localement et par touches. Ailleurs, les vernis sont épais, jaunis et dans l'ensemble très brillants, appliqués de façon régulière et dénaturant la palette chromatique de l'artiste autant que sa matière picturale (fig. 4) : il s'agit d'un geste de restaurateur, postérieur à l'exécution des œuvres. La décision de les enlever a été motivée par leurs qualités esthétiques médiocres, incompatibles avec la matière picturale originale et sans vernis dont témoigne une quinzaine de tableaux. Techniquement, ces vernis d'origine naturelle se sont avérés facilement réversibles dans des solvants volatils et peu polaires. Le nettoyage a conforté notre démarche de ne pas appliquer de nouveau vernis : l'aspect régulier, légèrement satiné, de la surface peinte, valorise la touche et la palette d'Helleu.
En ce qui concerne les repeints identifiés comme tels sur des lacunes, sur des accidents non mastiqués ou étendus sur le fond des compositions, ils ont été éliminés du mieux possible en fonction de leur réversibilité. Les manques ont ensuite été réintégrés chromatiquement de façon illusionniste. En revanche, les touches désaccordées suspectes ont fait l'objet de nombreuses interrogations, sans pouvoir apporter de réponse certaine. Le cas le plus flagrant concerne Alice Helleu lisant sur la plage de Deauville (inv. 2010.1.39, fig. 5), comme le révèle l'examen sous lumière ultraviolette ${ }^{9}$. De larges touches brun-jaune paraissent incongrues au milieu d'une palette harmonieuse de gris mauves. De même, Alice Helleu sur la plage de Deauville (inv. 2010.1.46) est parsemé de taches plus foncées, huileuses et, semble-t-il, contemporaines de la matière picturale. Celles-ci ont été conservées car elles ne réagissaient pas aux solvants des repeints déjà rencontrés, mais seulement à des produits risquant d'endommager l'original. Le caractère autographe de ces touches de couleurs, qui s'apparentent à des reprises locales, est-il plausible ? Paulette Howard-Johnston était elle-même peintre. La comparaison des teintes fréquemment utilisées par cette dernière avec celles employées pour ces repeints en souligne les similitudes. Est-elle intervenue sur les œuvres de son père ? Si ces touches semblent compatibles avec la gestuelle d'Helleu, elles restent posées sur le motif sans lien avec lui. La lecture des compositions s'en trouve perturbée. Cependant, enlever ces 


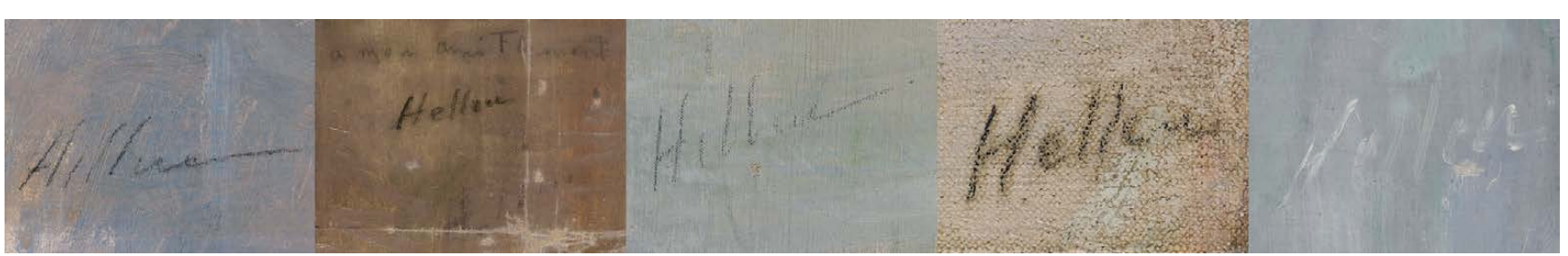

Fig. 6. Série de signatures apposées sur les œuvres d'Helleu.

De droite à gauche, détails de Fleurs dans une coupe, huile sur toile (37,3 x 50,8 cm) (inv. 2010.1.37), signature présumée autographe ; Vase d'hortensias sur un guéridon carré; Alice Helleu lisant sur la plage de Deauville; Ellen Helleu à la poupée; Bouquet de lilas dans un vase en argent, huile sur toile (81 x 64,9 cm) (inv. 2010.1.21). (C) Bayonne, musée Bonnat-Helleu/A. Vaquero.

repeints était radical et non sans danger. Compte tenu des doutes que nous avions et des difficultés à retirer cette matière huileuse épaisse, nous avons fait le choix de les conserver, en les atténuant par une retouche en glacis et en les harmonisant avec la matière picturale environnante, cette opération étant parfaitement réversible.

La question des reprises ultérieures se pose également avec les signatures. Leurs formes et techniques différentes, la comparaison avec Fleurs dans une coupe (inv. 2010.1.37, fig. 6) dont la couche picturale ne semble avoir subi aucune intervention et nous sert de référence, nous amènent à penser que bon nombre ne sont pas autographes. Parfois, la signature a été tracée deux fois, lorsqu'un changement de format vient occulter celle que l'on peut supposer être la plus ancienne. Vase d'hortensias sur un guéridon carré (inv. 2010.1.27, fig. 6) porte ainsi une signature à la peinture mauve sur le pli de la toile rabattue au dos du châssis, et une seconde à la pierre noire (?) sous la dédicace. Sur Alice Helleu lisant sur la plage de Deauville (inv. 2010.1.39, fig. 6), le nom de l'artiste est apposé sur des retouches. Il est également tracé sur le vernis, sûrement non original, d'Ellen Helleu à la poupée (inv. 2010.1.41, fig. 6). L'étude de Bouquet de lilas dans un vase en argent (inv. 2010.1.21, fig. 6) témoigne d'une reprise complète de la composition en raison d'accidents et d'un mauvais état général : la signature est présente sur une matière picturale opaque, de qualité médiocre. Pourtant, le repeint vert déborde sur quelques lettres sous-jacentes, laissant supposer qu'elle est originale... Enfin, la question de l'authenticité des signatures portées sur des œuvres manifestement inachevées reste ouverte. Aucune modification n'a été apportée à ces inscriptions dont l'histoire reste encore à préciser.

\section{Conclusion}

Les observations faites sur les peintures, confrontées les unes aux autres en prenant pour référence les 10 tableaux n'ayant assurément subi aucune restauration de la couche picturale ${ }^{10}$, ont permis de comprendre la technique de Paul Helleu et par conséquent d'identifier les éléments étrangers à sa pratique : touches décalées chromatiquement, vernis disharmonieux, signatures multiples. Il paraît ainsi évident que plusieurs tableaux de la collection ont subi des interventions, non seulement de conservation ou de restauration, mais aussi d'ordre esthétique. Des choix de traitements cohérents, que nous estimons respectueux et minimalistes, ont ainsi pu être opérés tout en préservant l'histoire de chacune des toiles. Les teintes subtiles de l'artiste ont été révélées par le nettoyage de la couche picturale, et l'allègement des vernis trop brillants a rendu aux œuvres leur légèreté et mis en évidence la touche du peintre. Malgré les interrogations qui subsistent, le fonds a retrouvé l'homogénéité qui lui manquait et sera exposé dans quelques années sur les cimaises du musée Bonnat-Helleu rénové.
Notes

1. $\mathrm{N}^{\circ} 128$ de la vente du 24 février 1943 à l'hôtel Drouot.

2. Blanche, 1928 , p. 123-124.

3. Flament, 1931.

4. Blanche, 1928, p. 140.

5. Goncourt de, 1896, 31 janvier 1895 , p. 301.

6. Toiles de $22 \times 22$ fils/cm jusqu'à 35 x 35 fils/cm pour les plus fines, quelques cas de toiles plus lâches (12 x 12 fils $/ \mathrm{cm})$.

7. Il s'agit de la nature morte Vase d'hortensias dans un panier tressé (inv. 2010.1.35).
8. Les œuvres rentoilées sont les suivantes : 2010.1.13, 2010.1.15, 2010.1.16, 2010.1.20, 2010.1.21, 2010.1.22, 2010.1.23, 2010.1.24, 2010.1.29, 2010.1.30, 2010.1.32, 2010.1.37, 2010.1.42, 2010.1.44, 2010.1.47, CM 557.

9. Il n'y a pas eu de dossier d'imagerie scientifique à proprement parler. Seules quelques photographies sous UV et en lumière rasante ont été prises par le photographe du musée Bonnat-Helleu pour mettre en évidence l'aspect de la couche picturale.

10. Les tableaux suivants ont été pris pour référence : 2010.1.17, 2010.1.19, 2010.1.22, 2010.1.25, 2010.1.29, 2010.1.34, 2010.1.37, 2010.1.43, 2010.1.47, 2010.1.239

\section{Bibliographie}

Blanche J.-É., 1928, « Souvenirs sur Helleu et le monde de sa jeunesse ", dans Propos de peintre, III. De Gauguin à la Revue Nègre, Émile-Paul Frères, Paris, p. 115149.

Flament A., 7 novembre 1931, « Helleu paysagiste ", L'Illustration, $\mathrm{n}^{\circ} 4627$, p. 328.

Goncourt de, E. et J., 1896, Journal des Goncourt. Mémoires de la vie littéraire, troisième série - troisième volume, tome IX. 1892-1895, G. Charpentier et E. Fasquelle, Paris. 